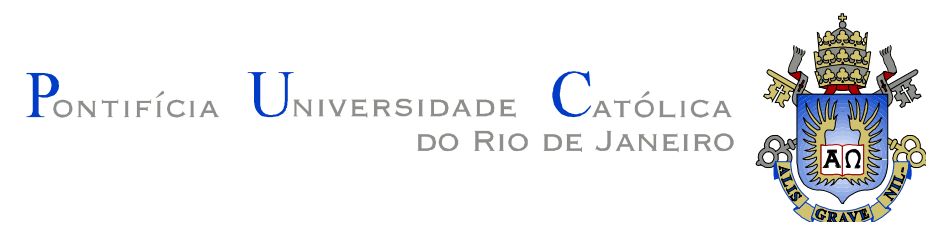

Rian Esteves Aderne

\title{
Desenvolvimento e caracterização de OLEDs baseados em sondas fluorescentes
}

Dissertação de Mestrado

Dissertação apresentada como requisito parcial para obtenção do título de Mestre pelo Programa de Pós-Graduação em Física da PUC-Rio.

Orientador: Prof. Marco Cremona 
Rian Esteves Aderne

\section{Desenvolvimento e caracterização de OLEDs baseados em sondas fluorescentes}

Dissertação apresentada como requisito parcial para obtenção do grau de Mestre pelo Programa de Pós-Graduação em Física do Departamento de Física do Centro Técnico Científico da PUC-Rio. Aprovada pela Comissão Examinadora abaixo assinada.

Prof. Marco Cremona

Orientador

Departamento de Física - PUC-Rio

Profa. Sônia Renaux Wanderley Louro Departamento de Física - PUC-Rio

Prof. Simon John Garden

UFRJ

Profa. Emy Niyama

Departamento de Física - PUC-Rio

Prof. José Eugenio Leal

Coordenador Setorial do Centro

Técnico Científico - PUC-Rio

Rio de Janeiro, 25 de setembro de 2013. 
Todos os direitos reservados. É proibida a reprodução total ou parcial do trabalho sem autorização da universidade, da autora e do orientador.

\section{Rian Esteves Aderne}

Bacharel em Física pela Universidade Federal do Rio de Janeiro (UFRJ, Rio de Janeiro-2010). Tem experiência na área de matéria condensada com ênfase em eletrônica orgânica.

Ficha Catalográfica

Aderne, Rian Esteves

Desenvolvimento e caracterização de OLEDs baseados em sondas fluorescentes./Rian Esteves Aderne; orientador: Marco Cremona. - 2013.

140: il.(color.) ; 29,7 cm

Dissertação (Mestrado) - Pontifícia Universidade Católica do Rio de Janeiro, Departamento de Física, 2013.

Inclui bibliografia.

1. Física - Teses. 2. Semicondutores orgânicos. 3. Eletrônica orgânica. 4. Filmes finos. 5. Transferência de energia. 6. Eletroluminescência. 7. Fotoluminescência. I. Cremona, Marco. II. Pontifícia Universidade Católica do Rio de Janeiro. Departamento de Física. IV. Título. 
"Sonda-me, ó Deus, e conhece o meu coração; prova-me, e conhece os meus pensamentos; vê se há em mim algum caminho perverso, e guia-me pelo caminho eterno."

Salmos, 139: 23 - 24. 


\section{Agradecimentos}

Ao DEUS Pai, nosso Salvador Jesus Cristo, nosso SENHOR;

A meus pais Ricardo e Elizabeth, e aos meus irmãos, Eric e Lucas, pelo apoio e incentivo;

A minha namorada Malu pelo: carinho, incentivo, apoio e compreensão pela minha ausência nos finais de semanas;

Ao Prof. Dr. Marco Cremona, pela sua valiosa e dedicada orientação durante o trabalho do mestrado, pela sua vasta experiência em OLEDs, por me fazer despertar para o pensamento crítico na pesquisa e pelo seu bom exemplo de vida profissional;

A Dra. Emy, pela dedicada assistência durante o desenvolvimento do trabalho de mestrado, desde as simples conversas no café à até importante ajuda na revisão deste trabalho;

A Dra. Carol do INMETRO pelo ensinamento e treinamento da técnica de voltametria cíclica;

Aos colaboradores deste trabalho Prof. Dr. Nicolás Rey e sua doutoranda Rafaella Santos, pela amostra de DMD; ao Prof. Dr. Fabiano Rodembush e à Prof. Dra. Leandra Campos pela amostra 5ONCS e ao Prof. Dr. Simon Garden e sua doutoranda Paola Gama pelas amostras de DIPI e TDIPI;

Ao laboratório LMSCP da COPPE UFRJ pelas medidas de TGA e DSC, e em especial as técnicas Grazi e Rhanayara pelas medições das minhas amostras e pelos esclarecimentos sobre as técnicas de medidas;

Aos meus colegas do laboratório, LOEM: Emy, Rafael, Kelly, Vanessa, Harold, Yolanda, Gustavo, Bia, Anderson e Pedro; e aos muitos amigos e alunos da pósgraduação que de certa forma torceram por mim;

Ao pessoal do Departamento de Física, pela ajuda de todos os dias;

Ao CNPq e FAPERJ pelo apoio financeiro, e à PUC-Rio pela utilização das instalações e laboratórios. 


\section{Resumo}

Aderne, Rian Esteves; Cremona, Marco. Desenvolvimento e caracterização de OLEDs baseados em sondas fluorescentes. Rio de Janeiro, 2013. 140p. Dissertação de Mestrado - Departamento de Física, Pontifícia Universidade Católica do Rio de Janeiro.

Nesta dissertação foram estudadas as propriedades ópticas, eletroquímicas, elétricas e morfológicas de novos compostos fluorescentes para o desenvolvimento de OLEDs. Para isto, foram estudadas algumas sondas moleculares fluorescentes utilizadas na área biomédica como agentes antitumorais e marcadores ópticos fluorescentes: a) N,N'- diisonicotinoil-2-hidroxi-5 metilisoftaldeído diidrazona (DMD); b) 2-(5'-isotiocianato-2'hidroxifenil)benzoxazol, (5ONCS); c) 1,1'-dipireno (DIPI) e d) 7,7'-terc-butil-1,1'dipireno (TDIPI). Todos estes compostos foram sintetizados por Grupos de pesquisa brasileiros e depositados termicamente em forma de filmes finos no nosso Laboratório. No decorrer do estudo de fabricação dos OLEDs, os dispositivos bicamada baseados no DMD e no 5ONCS se mostraram pouco eficientes devido principalmente à baixa condutividade do DMD e à elevada rugosidade da camada de 5 ONCS. A solução destes problemas foi encontrada na técnica de codeposição, que consiste na evaporação simultânea de uma matriz orgânica e de um dopante (DMD ou 5ONCS) numa única camada. Desta forma, foi possível alcançar um aumento da mobilidade das cargas nas camadas co-depositadas alem de favorecer a transferência de energia da matriz para o dopante. Os OLEDs fabricados nestas condições permitiram observar, pela primeira vez, a eletroluminescência dos compostos DMD e 5ONCS. Já os OLEDs baseados nas moléculas DIPI e TDIPI apresentaram eletroluminescência sem a necessidade da co-deposição. Em particular, no caso do OLED baseado no TDIPI foi possível alcançar uma luminância de $1430 \mathrm{~cd} / \mathrm{m}^{2} \mathrm{com}$ uma eficiência de 2,65\% a $1 \mathrm{~mA}$. Os resultados deste trabalho evidenciam a potencialidade do uso destes materiais para a fabricação de OLEDs para aplicações na área de iluminação.

\section{Palavras Chave}

OLEDs; codeposição; sondas fluorescentes; filmes finos; transferência de energia; eletroluminescência; Eletrônica Orgânica; semicondutores orgânicos. 


\section{Abstract}

Aderne, Rian Esteves; Cremona, Marco (Advisor). Development and characterization of OLEDs based on fluorescent probes. Rio de Janeiro, 2013. 140p. MSc. Dissertation - Departamento de Física, Pontifícia Universidade Católica do Rio de Janeiro.

In this study the optical, electrochemical, electrical and morphological properties of new fluorescent compounds were studied in order to develop OLEDs based upon these materials. For this purpose some fluorescent molecular probes used in the biomedical field as antitumor agents and fluorescent optical probes were studied: a) N,N' diisonicotinoyl-2-hydroxy-5methylisophthalaldehyde dihydrazone (DMD ), b) 2 - (5' -isothiocyanato -2'hydroxyphenyl) benzoxazole ( 5ONCS ), c) 1,1'- dipyrene (DIPI) and d) 7,7'-tertbutyl-1,1'-dipyrene (TDIPI ). All these compounds were synthesized by Brazilian research groups and then thermally deposited as thin films in our Laboratory. During the study for the fabrication of OLEDs, bilayer devices based on DMD and 5 ONCS proved to have low efficiency mainly due to the low conductivity of the DMD and the high roughness of the 5ONCS layer. The solution of these problems was found in the codeposition technique, which consists in the simultaneous evaporation of an organic matrix (host) and a dopant (guest) (5ONCS or DMD) in a single layer. Thus, it was possible to achieve an increase in the charge mobility in the co-deposited layers as well as energy transfer from the guest to the host. The OLEDs fabricated in these conditions allowed the observation, for the first time, of the electroluminescence of DMD and 5ONCS. On the other hand, the DIPI and TDIPI based OLEDs presented good electroluminescence without the need for co-deposition. In particular, in the case of the TDIPI it was possible to achieve a luminance of $1430 \mathrm{~cd} / \mathrm{m}^{2}$ with an efficiency of $2.65 \%$ at $1 \mathrm{~mA}$. The results of this work showed the potential of these materials for the fabrication of OLEDs for lighting applications

\section{Keywords}

OLEDs; codeposition; fluorescent probes; thin films; energy transfer; electroluminescence; organic electronics; organic semiconductors. 


\section{Sumário}

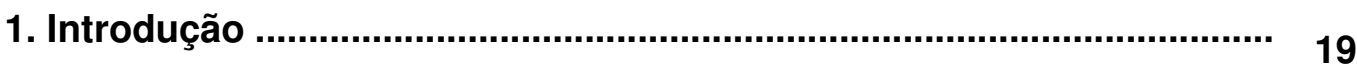

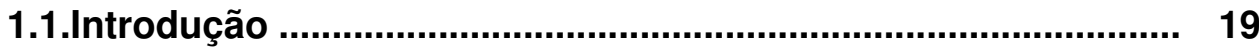

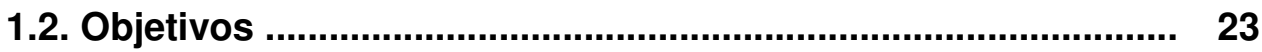

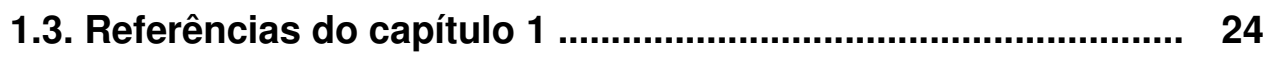

2. Fundamentos teóricos ................................................................... 25

2.1. Sondas moleculares fluorescentes ....................................... 25

2.1.1. Família das Hidrazonas ........................................ 25

2.1.2. Família das Benzazolas ....................................... 26

2.1.3. Familia dos Pirenos .............................................. $\quad 28$

2.2. Luminescência ........................................................................ 28

2.2.1. Fotoluminescência .............................................. 29

2.2.2. Eletroluminescência ........................................... 32

2.3. Filmes Finos ....................................................................... 33

2.3.1. Técnicas de deposição ........................................... 34

2.3.2. Técnica de co-deposição térmica .......................... 35

2.3.2.1.Transferência de energia ......................... 37

2.4. OLEDs ................................................................................... 39

2.4.1. Mecanismos de funcionamento de um OLED ....... $\quad 40$

2.5. Determinação dos níveis HOMO e LUMO .............................. 47

2.5.1. Obtenção dos parâmetros eletroquímicos ............. 47

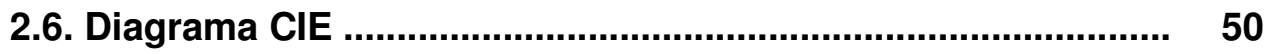

2.7. Eficiência dos OLEDs ......................................................... 53

2.8. Referência capítulo 2 ........................................................... 54

3. Materiais e Métodos ....................................................................... 59 
3.1. Materiais utilizados ........................................................ $\quad 59$

3.1.1. Sondas moleculares fluorescentes ..................... $\quad 59$

3.1.2. Demais materiais utilizados .................................. 62

3.2. Procedimentos experimentais ............................................. 64

3.2.1. Limpeza de substratos ....................................... 65

3.2.2. Evaporação térmica resistiva ............................... 66

3.3. Técnicas de caracterização .................................................. 68

3.3.1. Perfilômetria .............................................. 68

3.3.2. Análise Térmica ................................................. 70

3.3.2.1. Análise termogravimétrica (TGA / DTG) .. 71

3.3.2.2. Calorimetria diferencial de varredura 72 (DSC)

3.3.3. Determinação dos níveis HOMO e LUMO .............. 73

3.3.3.1. Voltametria Cíclica (VC) .......................... 74

3.3.3.2. Espectroscopia de Absorção UV-Vis ..... 77

3.3.4. Espectroscopia de Fotoluminescência ................. 78

3.3.5. Espectroscopia de Eletroluminescência ............... 79

3.3.6. Medidas de luminância ........................................ 80

3.3.7. Caracterização elétrica ....................................... 81

3.4. Referências do capítulo 3 ...................................................... 82

4. Resultados e discussão .................................................................. 85

4.1. Caracterização das sondas moleculares fluorescentes ...... 85

4.1.1. Caracterização térmica dos compostos estudados 85

4.2. Determinação dos níveis de HOMO e de LUMO ................... 89

4.3. Perfilômetria ............................................................... 92

4.4. Estudos das propriedades ópticas e elétricas do $\mathrm{DMD}^{1} \ldots . . \quad 93$

4.4.1. Fotoluminescência .................................................. 93

${ }^{1} \mathrm{~N}, \mathrm{~N}$ '- diisonicotinoil-2-hidroxi-5 metilisoftaldeído diidrazona 
4.4.2. Eletroluminescência ............................................ 94

4.4.2.1. Codeposição matriz :DMD ....................... 94

4.4.2.2. OLEDs baseados no DMD ....................... 98

4.4.2.3. Estudo das concentrações de dopante 100 nos dispositivos

4.5. Estudos das propriedades ópticas e elétricas do composto 104 50 NCS $^{2}$

4.5.1 Fotoluminescência .................................................. 104

4.5.2 Eletroluminescência ......................................................... 105

4.5.2.1. Codeposição de filmes de 5ONCS ........... 105

4.5.2.2. OLEDs de filmes codepositados ............ 109

4.6. Estudos das propriedades ópticas e elétricas do Dipireno 115 (DIPI ${ }^{3}$ e TDIPI ${ }^{4}$ )

4.6.1. Fotoluminescência ................................................ 115

4.6.2. Eletroluminescência .............................................. 116

4.7. Regime de transporte de cargas .................................... 122

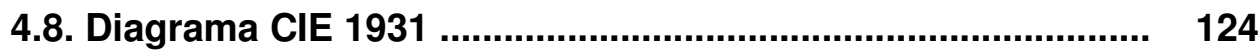

4.9. Referência do capítulo 4 ...................................................... 126

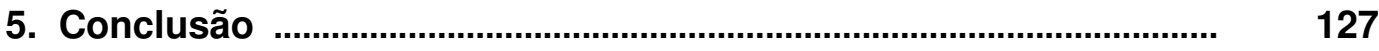

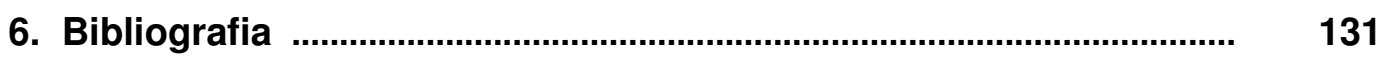

2 2-(5'-isotiocianato-2'-hidroxifenil)benzoxazol

${ }^{3} 1,1^{\prime}$ - dipireno

${ }^{4} 7,7$ '-terc-butil-1,1'-dipireno 


\section{Lista de Figuras}

Figura 1.1 a. Pentaceno (molécula orgânica) e b. Polipirrol (polímero).

Figura 1.2 Produtos já comercializados que utilizam a tecnologia OLED a. 22 Smartphone Samsung Galaxy S IV b. SmartTV LG em substratos curvos.

Figura 2.1 Estrutura molecular de uma hidrazona.

Figura 2.2 Estrutura de um 2-(2-hidroxifenil)benzoxazol.

Figura 2.3 Mecanismo ESIPT na molécula 2-(5'-isotiocianato-2'-

hidroxifenil) benzoxazol (5ONCS).

Figura 2.4 Estrutura molecular do pireno.

Figura 2.5 Diagrama de Jablonski apresentando as transições eletrônicas possíveis, quando uma molécula absorve energia.

Figura 2.6 a. Representação dos espectros de emissão (Ems) e de 36 absorção (Abs) do conjunto matriz:dopante numa camada codepositada. Em amarelo a região de sobreposição que permite a transferência de energia entre a matriz e dopante; b. Digrama de Jablonski com a representação da transferência de energia.

Figura 2.7 Exemplo esquemático da codeposição com ênfase para a posição dos cristais de detecção de espessura e dos cadinhos da matriz(M) e dopante(D).

Figura 2.8 Arquiteturas comuns em OLEDs - a. Bicamada e b. Multicamada.

Figura 2.9 Os mecanismos de eletroluminescência em OLEDs.

Figura 2.10 Esquema dos processos de injeção termiônica e injeção por tunelamento em uma estrutura metal/semicondutor-intrínseco/metal. $\varphi$ corresponde à altura da barreira de potencial na interface metal/semicondutor e $\mathrm{V}$ ao potencial elétrico aplicado entre os eletrodos. 
Figura 2.11 Comportamento característico para o gráfico $\ln \left(\mathrm{I} / \mathrm{E}^{2}\right) \times(1 / \mathrm{E})$,

onde E é campo elétrico aplicado. Um ajuste para teoria Fowler-Nordheim (linha continua) para injeção por tunelamento é apresentado.

Figura 2.12 Comportamento característico da corrente para um dispositivo orgânico onde a injeção termiônica governa a injeção de portadores (linha continua). Inserida na figura é apresentado o comportamento do logaritmo da corrente em função do inverso da T.

Figura 2.13 a. Comportamento da densidade de corrente em função do campo elétrico aplicado para o funcionamento de um OLED e b. regimes de transporte para o funcionamento de um OLED.

Figura 2.14 Processos de formação de éxciton, de recombinação e de emissão num dispositivo OLED.

Figura 2.15 Diagrama de bandas para uma estrutura metal/orgânico/metal.

Figura 2.16 Exemplo de um voltamograma, com ênfase nos potenciais de oxidação e redução e nos processos de oxidação e de redução.

Figura 2.17 Curvas padrões para as cores primárias do CIE.

Figura 2.18 Diagrama de cromaticidade CIE em 1931.

Figura 3.1 Estrutura molecular N,N'- diisonicotinoil-2-hidroxi-5 metilisoftaldeído diidrazona (DMD).

Figura 3.2 Estrutura da molecular 2-(5'-isotiocianato-2'hidroxifenil)benzoxazol (5ONCS).

Figura 3.3 Estrutura molecular de: a. 1,1'-dipireno (DIPI) e b. 7,7'-tercbutil-1,1'-dipireno (TDIPI).

Figura 3.4 Representação da estrutura do 1,1'-dipireno a. ângulo entre os planos dos anéis de $72,11^{\circ}$ (torção) e $\mathbf{b}$. ângulo entre os planos dos anéis de $115.64^{\circ}$.

Figura 3.5 Estruturas moleculares dos transportadores de cargas ( $\beta$-NBP, $\mathrm{MTCD}, \mathrm{Alq}_{3}$ ), das matrizes (BSBF e BSB) e do bloqueador de elétrons (BCP).

Figura 3.6 a. Substratos de vidro recoberto com camada de ITO e b. Representação das áreas ativas dos OLEDs fabricados.

Figura 3.7 a. Foto do sistema de deposição UNIVEX 300 e b. as 5 posições de deposição disponíveis no sistema. 
Figura 3.8 a. llustração dos cadinhos usados para a evaporação dos compostos orgânicos. Em b. Figura do cadinho (basket) de Tungstênio usado para deposição de Al.

Figura 3.9 a. Perfilômetro Veeco DekTak 150 com acessório "low force option" b. esquema de medida de uma amostra; c. gráfico obtido com o perfilômetro, nota-se o degrau de 50nm.

Figura 3.10 Exemplo de medida de TGA / DTG para o $\mathrm{Alq}_{3}$.

Figura 3.11 Esboço teórico de uma curva DSC com as principais temperaturas de transição observáveis: $T_{g}$ - temperatura de transição vítrea, $T_{c}$ - temperatura de cristalização e $T_{m}$ - ponto de fusão.

Figura 3.12 Método para se determinar a $T_{g}$ em uma curva de DSC.

Figura 3.13 Representação esquemática da disposição dos eletrodos na célula eletrolítica.

Figura 3.14 Curvas de voltametria cíclicas correspondentes ao Ferroceno (FC) e ao composto investigado DIPI (1,1'- dipireno).

Figura 3.15 Exemplo de um espectro de absorção coma indicação de comprimento de onda $\lambda_{g}$, do gap. Em detalhe o diagrama dos níveis de energia HOMO e LUMO, cuja diferença resulta no valor de gap.

Figura 3.16 Representação esquemática do espectrofluorímetro da PTI: 1. Lâmpada de arco de Xe 150 Watts; 2. Fendas ajustáveis; 3. Modulo monocromador de excitação; 4. Câmara da amostra; 5. Lentes para focalização; 6. Suporte para a amostra; 7. Modulo monocromador de emissão; 8. Fotomultiplicadora.

Figura 3.17 a. Representação do espectrofluorímetro para medidas de Eletroluminescência e b. detalhe do porta-amostra para OLEDs.

Figura 3.18 Representação do sistema de medição de luminância.

Figura 4.1 Termogramas do DMD: a. Curvas TGA / DTG e b. curvas DSC e derivada e c. ampliação da região de $140^{\circ}$ a $180^{\circ} \mathrm{C}$ para a determinação 86 da $T_{g}$.

Figura 4.2 Termogramas do 5ONCS: a. Curvas TGA / DTG, b. curvas DSC e derivada e c. determinação do $T_{g}$.

Figura 4.3 Termogramas TGA e DTG a. para o DIPI b. para o TDIPI.

Figura 4.4 Medidas de voltametria cíclica (voltamogramas) dos compostos investigados a. DMD, b. 5ONCS, c. DIPI e d. TDIPI. 
Figura 4.5 Espectro de absorção dos compostos investigados a. DMD, b.

$50 N C S$, c. DIPI e d. TDIPI. As figuras inseridas evidenciam a

determinação do gap.

Figura 4.6 Diagrama rígido das bandas de energia para os compostos estudados.

Figura 4.7 Espectro PL do filme de DMD.

Figura 4.8 Espectro de absorção e de emissão da matriz BSBF.

Figura 4.9 Espectros de emissão do BSBF (1) doador e absorção do

DMD (2) receptor, a região em amarelo representa a sobreposição dos

96 espectros.

Figura 4.10 a. Espectros de absorção dos filmes de: DMD, BSBF, codepositado BSBF: DMD 25\% b. Espectro de fotoluminescência do filme de DMD c. Espectro de fotoluminescência do filme co-depositado BSBF:DMD 25\%.

Figura 4.11 a. Espectro EL para o Dispositivo 3 em função da tensão 98 aplicada. b. Espectro de EL do Dispositivo 3 e de $\mathrm{PL}$ dos filmes de $\mathrm{Alq}_{3} \mathrm{e}$ DMD.

Figura 4.12 a. Espectro de EL do Dispositivo 4, para tensões de 15V e 99 20V. b. Espectro de EL do Dispositivo 2 (1) e de PL do $\mathrm{Alq}_{3}(2)$ e do DMD (3).

Figura 4.13 Diagrama rígido de bandas de energia em função da espessura: a. para o Dispositivo 4 e b. para a camada codepositada 100 BSBF:DMD.

Figura 4.14 Espectros de EL: a. Dispositivo 5, b. Dispositivo 4, c. Dispositivo 6 e d. Dispositivo 7.

Figura 4.15 Relação das intensidades em 590nm em função das concentrações de DMD.

Figura 4.16 Curvas de luminância para o Dispositivo 6 com dopagem de $35 \%$ de DMD.

Figura 4.17 Espectro de (a.) absorção e de (b.) fotoluminescência de um filme de 5ONCS com 40nm de espessura.

Figura 4.18 Espectros de absorção e emissão dos compostos a. BSB e b. BSBF, inserida nas figuras a estruturas das moléculas.

Figura 4.19 a. Espectro de absorção e emissão dos compostos 5ONCS (2) e espectro de emissão da matriz BSB (1) b. Espectro de absorção do 
dopante 5ONCS (2) e espectro de emissão da matriz BSBF (1).

Figura 4.20 a. Espectro de absorção de filmes de BSB, 5ONCS e

BSB:5ONCS $30 \%$ b. Espectro de absorção de filmes de BSBF, 50 NCS e BSBF:5ONCS 30\%.

Figura 4.21 Espectro de PL do filme de a. BSB:5ONCS 30\% e b. BSBF:5ONCS $30 \%$.

Figura 4.22 Comparação entre o espectro de PL do filme 5ONCS e o do filme codepositado BSBF:5ONCS 30\%.

Figura 4.23 a. Espectro de EL do Dispositivo 9, b. Comparação entre os espectro de EL em 17V do dispositivo com os espectros de PL do BSB, $\beta$ NPB e 5ONCS.

Figura 4.24 a. Diagrama rígido de bandas de energia do Dispositivo 9 b. Níveis de energia da camada codepositada.

Figura 4.25 a. Espectro de eletroluminescência do Dispositivo 10 b. Comparação entre o espectro de EL do Dispositivo 10 e os espectros de PL do $\beta$-NPB, $\mathrm{Alq}_{3}$ e do filme codepositado BSBF:5ONCS 30\%.

Figura 4.26 a. Diagrama de bandas de energia do Dispositivo 10 b. Níveis de energia da camada codepositada de BSBF:5ONCS 30\%.

Figura 4.27 a. Espectros EL do Dispositivo 11 com variação de corrente e a figura inserida exibe o OLED de 5 ONCS em funcionamento $\mathbf{b}$. Comparação entre o espectro EL com PL do filme codepositado.

Figura 4.28 a. Curva de luminância do Dispositivo 11 b. da esquerda a direita, fotos o OLED desligado, a 5volts, 7Volts, 9Volts e 11Volts.

Figura 4.29 Espectros de PL de um filme de DIPI de 40nm de espessura depositado termicamente sobre um substrato de quartzo.

Figura 4.30 Espectro de PL de um filme de TDIPI de 40nm de espessura sobre um substrato de quartzo.

Figura 4.31 Espectro da eletroluminescência do Dispositivo 13 e uma foto inserida do OLED em funcionamento.

Figura 4.32 Comparação entre os espectros de PL dos compostos utilizados com o espectro de EL do Dispositivo 13.

Figura 4.33 Diagrama rígido de bandas de energia do OLED de DIPI.

Figura 4.34 Espectro de EL do Dispositivo 14. Na figura inserida uma foto do OLED em funcionamento.

Figura 4.35 Comparação entre os espectros de PL dos compostos com o espectro de EL do Dispositivo 14. 
Figura 4.36 Diagrama rígido de bandas de energia para o Dispositivo 14.

Figura 4.37 Luminescência do Dispositivo 14.

Figura 4.38 Gráficos V - I na escala log-log a. Dispositivo 5 b. Dispositivo

11 c. Dispositivo 13 e d. Dispositivo 14. Os ajustes lineares identificam os diferentes regimes de transporte de carga.

Figura 4.39 Gráfico de injeção por tunelamento para a. Dispositivo 5 b.

Dispositivo 11 c. Dispositivo 13 e d. Dispositivo 14.

Figura 4.40 a. Coordenadas das emissões dos compostos estudados $\mathbf{b}$.

coordenadas dos dispositivos fabricados para cada um dos compostos e

c. fotos dos dispositivos produzidos (i. Dispositivo 5 ii. Dispositivo 11 iii.

Dispositivo 13 e iv. Dispositivo 14). 


\section{Lista de tabelas}

Tabela 2.1-- Classificação das fontes de excitação de

luminescência

Tabela 3.1 -Compostos para a fabricação dos OLEDs 63

Tabela 4.1 -- Resultados da caracterização térmica 89

Tabela 4.2 -- Valores dos níveis de energia dos compostos 91

Tabela 4.3 -- Valores de TF e Fc para os compostos 92 investigados.

Tabela 4.4 - Coordenadas CIE dos compostos e dos OLEDs 125 fabricados 


\title{
Lista de abreviaturas
}

\author{
$A=$ Ampere \\ $\mathrm{Al}=$ alumínio \\ $\mathrm{Alq}_{3}=$ tris (8-hidroxiquinolina) de alumínio \\ $\mathrm{BC}=$ Banda de Condução \\ $\mathrm{BCP}=2,9$-dimetil-4,7 difenil-1,10 fenantrolina \\ $\mathrm{BSB}=4,4$ '-di (trifenilsililo)-bifenil \\ BSBF $=2$ - (9,9-spirobifluoreno-2-il)-9,9- spirobifluoreno \\ $\mathrm{BV}=$ Banda de Valência \\ $\beta$-NPB $=n, n$ '-bis(naftaleno-2-il)-n,n'-bis(fenil)-bendizina \\ $\mathrm{CBB}=$ camada bloqueadora de buracos \\ $\mathrm{CBE}=$ camada bloqueadora de elétrons \\ $\mathrm{CE}=$ camada emissora \\ $\mathrm{CIS}=$ Cruzamento intersistema \\ CTB = camada transportadora de buracos \\ CTE = camada transportadora de elétrons \\ DSC $=$ Calorimetria diferencial de varredura \\ DTG = Derivada da curva termogravimétrica \\ $E L=$ Eletroluminescência \\ $\mathrm{Fc}=$ fator de correção \\ $\mathrm{FC}=$ Ferroceno \\ $\mathrm{HOMO}=$ Orbital molecular mais alto ocupado \\ IP = Potencial de lonização \\ ITO = Óxido de índio dopado com estanho \\ LOEM = Laboratório de optoeletrônica molecular \\ LUMO = Orbital molecular mais baixo desocupado \\ LUMTEC = Luminescence Technology Corporation \\ OLED = Dispositivo orgânico emissor de luz \\ $\mathrm{PL}=$ Fotoluminescência \\ PVD = Physical vapor deposition \\ SCLC $=$ Corrente limitada por carga espacial \\ TGA = Termogravimétrica \\ $\mathrm{T}_{\mathrm{g}}=$ Temperatura vítrea \\ $\mathrm{TCL}=$ Corrente limitada por carga aprisionada \\ $\mathrm{TF}=$ Tooling Factor \\ UV-Vis = Ultravioleta - visível
}


$\mathrm{V}=$ Volts

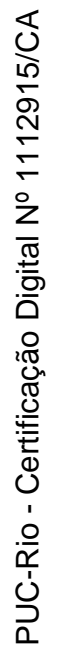

